\title{
Force Control of Electrical Load System Based on Single Neuron PID Adaptive and Repetitive Control
}

\author{
Zhiqiang Wei, Guanghua Zong, Hongchong Wu \\ Robotics Institute \\ Beihang University \\ Beijing, China \\ wzq761229@sohu.com
}

\begin{abstract}
In view of the surplus torque and complexity of controlled plant in passive electrical load system, a novel approach based on single neuron PID adaptive control and repetitive control for repetitive periodic load control system is proposed. Radial basis function (RBF) neural network is used to identify the system on-line for the single neuron PID controller to adjust its weights and PID parameters by selflearning and self-adapting based on the desired output. The dynamic state performance can be improved by the single neuron adaptive PID control and the steady state performance is also improved by modified repetitive control. Computer simulation results show that the force/position hybrid control system can effectively reduce the surplus torque and improve the loading precision, and also it has fine dynamic and steady state performance and good robustness. The reliability of whole system is further improved.
\end{abstract}

Keywords-surplus torque; electrical load system; single neuron; PID control; repetitive control

\section{INTRODUCTION}

The electrical load system(ELS) is an important equipment of the hardware-in-loop simulation, which is more and more widely used to test the performance of new aircraft rudder. ELS is a torque servo control system connected with rudder rigidly, so the motion of rudder will lead to surplus torque which is seriously affect the performance of control system and dynamic loading precision[1-2]. However, it is difficult to establish exact mathematical model for the ELS to realize high precision loading control since it has several disadvantages such as friction, the gap, the strong structure coupling between rudder and the loading motor, the time-varying parameters and so on. So the key problem of ELS is to eliminate the surplus torque with traditional method or modern intelligent control algorithm.

Single neuron PID adaptive controller has abilities of self-adapting and self-learning and nonlinear reflection as well as good robustness and its simple structure. Radial basis function neural network (RBFNN) is a kind of threelayer feedforward neural network with single hidden layer, it has the fast convergence speed and small calculating amount, and also it can avoid getting stuck in local minimum point. Meanwhile, it has been demonstrated that it can approximate arbitrary continuous function with arbitrary precision, so it is widely used in the nonlinear and uncertain control system [3-4].

Repetitive control based on internal model principle is very efficient to track periodic signal or restrain disturbance. Because of its high control precision and easy implementation as well as control performance independent of parameters, it has become one kind of valid means to resolve control problem for periodic extrinsic signal.

The performance of the load equipment is often tested by the sinusoidal torque signal, and so a integrated control strategy based on RBF on-line identification for single neuron PID adaptive controller and modified repetitive controller is proposed. The good characteristics of both neural networks and repetitive controller are used in the torque servo system so that both the control performance and loading precision can be improved. Simulation results show that the method can effectively raise the force servo performance and reduce the surplus torque, moreover the system has good ability to restrain disturbance and can guarantee the loading precision.

\section{Single NeURON PID CONTROL BASED ON RBFNN} A. Adaptive control based on neural network

Fig.1 is the structure diagram of single neuron PID adaptive control system based on RBFNN[5-6].

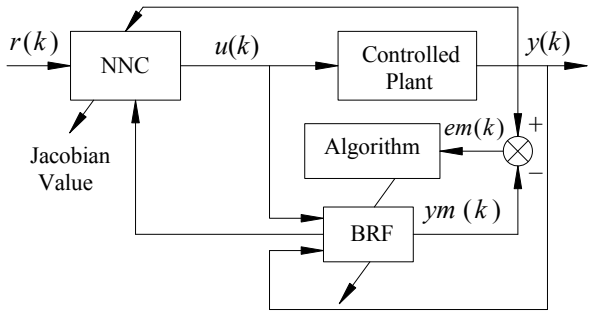

Fig.1. Single neuron PID adaptive control system based on RBF

NNC is implemented by single neuron PID controller, and RBF is the neural network identifier(NNI) to identify the Jacobian value of the controlled plant. $r(k)$ is the system input, $y(k)$ and $u(k)$ are the inputs of NNI, $y(k)$ is the actual output, $y m(k)$ is the output of RBF.The output of NNI can approximate actual system output effectively when the algorithm is steady and after identifying and learning, i.e., $y m(k) \approx y(k)$.

\section{B. The RBFNN model}


Fig.2.

$\mathrm{RBF}$ is a kind of three-layer neural network as shown in

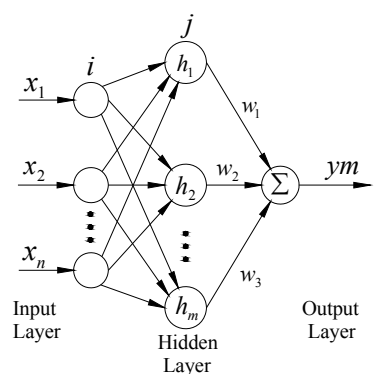

Fig.2. Structure diagram of RBF model

Where $X=\left[x_{1}, x_{2}, \ldots, x_{n}\right]^{T}$ is the network input vector, and $H=\left[h_{1}, h_{2}, \ldots, h_{j}, \ldots, h_{n}\right]^{T}$ is the radial basis vector of RBFNN, the Gaussian function $h_{j}$ is

$$
h_{j}=\exp \left(-\frac{\left\|X-C_{j}\right\|}{2 b_{j}^{2}}\right), j=1,2, \ldots m
$$

The jth node central vector is

$$
C_{j}=\left[c_{j 1}, c_{j 2}, \ldots, c_{j i}, \ldots, c_{j n}\right]^{T}, i=1,2, \ldots n
$$

assume that he basis width vector is $B=\left[b_{1}, b_{2}, \ldots, b_{j}, \ldots, b_{m}\right]^{T}, b_{j}$ is the parameter of node $j$ basis width and it is greater than zero, the network weight vector is

$$
W=\left[w_{1}, w_{2}, \ldots, w_{j}, \ldots, w_{m}\right]^{T}
$$

the output of NNI is

$$
y m(k)=w_{1} h_{1}+w_{2} h_{2}+\ldots+w_{m} h_{m}
$$

the index of identification is

$$
\operatorname{Em}(k)=\frac{1}{2}(y(k)-y m(k))^{2}
$$

according to the gradient descent method, the parameters of output weight, node centre, and the node basis width are updated as follows

$$
\begin{gathered}
w_{j}(k)=w_{j}(k-1)+\eta(y(k)-y m(k)) h_{j} \\
+\alpha\left(w_{j}(k-1)-w_{j}(k-2)\right) \\
\Delta b_{j}=(y(k)-y m(k)) w_{j} h_{j} \frac{\left\|X-C_{j}\right\|^{2}}{b_{j}^{3}} \\
b_{j}(k)=b_{j}(k-1)+\eta \Delta b_{j}+\alpha\left(b_{j}(k-1)-b_{j}(k-2)\right)(8) \\
\Delta c_{j i}=(y(k)-y m(k)) w_{j} \frac{x_{j}-c_{j i}}{b_{j}^{2}} \\
c_{j i}(k)=c_{j i}(k-1)+\eta \Delta c_{j i}+\alpha\left(c_{j i}(k-1)-c_{j i}(k-2)\right)(10)
\end{gathered}
$$

where $\eta$ is a learning rate, $\alpha$ is a momentum factor.

The Jacobian value, i.e., the sensitivity is shown below

$$
\frac{\partial y(k)}{\partial u(k)} \approx \frac{\partial y m(k)}{\partial u(k)}=\sum_{j=1}^{m} w_{j} h_{j} \frac{c_{j i}-x_{1}}{b_{j}^{2}}
$$

where, $x_{1}=u(k)$.

\section{The structure of single neuron PID controller}

Fig.3 is the structure diagram of single neuron PID adaptive controller, $w_{i}(k)$ is the weight vector of input neuron, $r(k)$ is system input, $y(k)$ is the system actual output, and $K_{u}$ is a proportion factor.

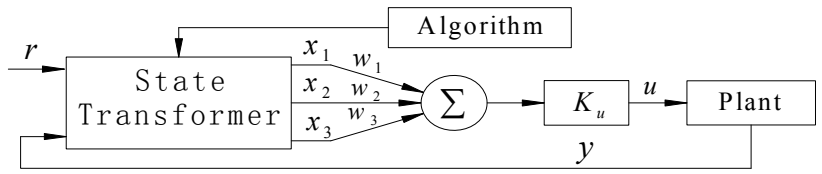

Fig.3. Structure of single neuron PID controller

The mathematical model of the single neuron PID adaptive controller is given below

$$
\begin{gathered}
x_{1}(k)=e c(k) \\
x_{2}(k)=T \sum_{k=1}^{k} e c(k) \\
x_{3}(k)=(e c(k)-e c(k-1)) / T
\end{gathered}
$$

where, $e c(k)=r(k)-y(k)$ is the error training signal for single neuron PID adaptive controller and its output is

$$
u(k)=K_{u} \sum_{j=1}^{3} w_{j}(k) x_{j}(k)
$$

The learning rule of supervised Delta algorithm is adopted by the single neuron PID adaptive controller

$$
\begin{aligned}
& E c=e c(k)^{2} / 2=(y r(k)-y(k))^{2} / 2 \\
& w_{i}(k+1)=w_{i}(k)+\eta_{i} e c(k) \frac{\partial y}{\partial u} x_{i}(k)
\end{aligned}
$$

where $\eta_{i}$ is the learning rate of single neuron, $\eta_{i}>0(i=1,2,3)$.

\section{Design of Modified Repetitive Controller}

A modified repetitive controller, which is shown in Fig.4, is adopted [7-8]. The error convergence speed and stability of system are both considered. In Fig.4, $r(s)$ is input signal with period $L, T_{s}$ is the sampling time, $F(s)$ is a low-pass filter, $K(s)$ is a compensator and $G(s)$ is the controlled plant.

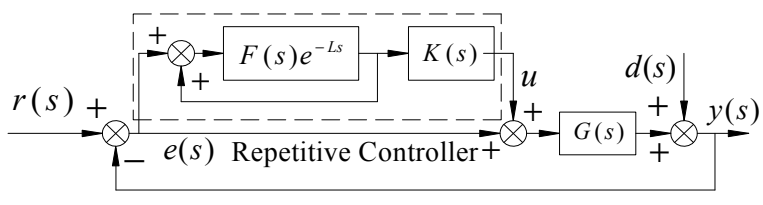

Fig.4. Structure of modified repetitive controller

From Fig.4, we have

$$
e(s)=\frac{r(s)-d(s)}{1+G(s)} \cdot \frac{1-F(s) e^{-L s}}{1-\left[1-G_{c}(s)\right] F(s) e^{-L s}}
$$

where, $G_{c}(s)=\frac{K(S) G(S)}{1+G(S)}$.

According to the small gain theorem and suppose that all the elements of input are bounded, the asymptotic stability condition of the system is

1) $G_{c}(s)$ is asymptotically stable. 


\section{2) $\left\|F(s)\left[1-G_{c}(s)\right]\right\|_{\infty}<1$.}

Here we let $F(s)=1 /(1+0.1 s)$ in the system so that the system not only can guarantee small error and restrain high frequency disturbance, but also can relax the stability conditions for the repetitive system. Yet since the low-pass filter is introduced, the system has some steady-state error, so compensator $K(s)$ is used to quicken error convergence speed and decrease the steady-state error, here $K(s)=0.05$.

\section{Mathematical Model of ELS}

The essential structure of the ELS is show in Fig.5. For the sake of analyzing convenience, some conditions is supposed as below $[1,9]$

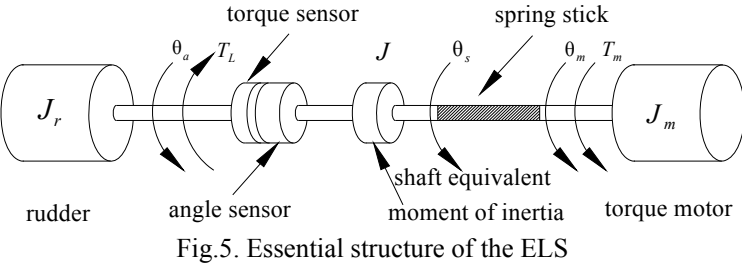

1) The connection between the motor and rudder is rigid.

2) The spring stick is a torque buffer to eliminate the high frequency noises of the load system and it can reduce the disturbance torque. Here, ignoring the spring stick's own mass, distortion of other parts of axis.

The torque by torque motor is proportional to the rotary angle difference of rudder and the motor. So the torque can be described as follows

$$
T_{L}=K_{L}\left(\theta_{m}-\theta_{a}\right)
$$

where $T_{L}$ is the load torque, Nm; $K_{L}$ is the stiffness coefficient of the spring stick, $\mathrm{Nm} / \mathrm{rad} ; \theta_{m}$ is the rotary angle of the torque motor, rad; $\theta_{s}$ is the rotary angle of the rudder, $\mathrm{rad}$, here $\theta_{a}=\theta_{s} ; T_{m}$ is the output torque of the motor, Nm.

According to the mechanism of the ELS, we can easily deduce the dynamic model, the equilibrium equations of control voltage $U_{m}$ and electromagnetic torque $T_{m}$ by the motor are described as (20), and the dynamic model of the ELS is shown as Fig.6.

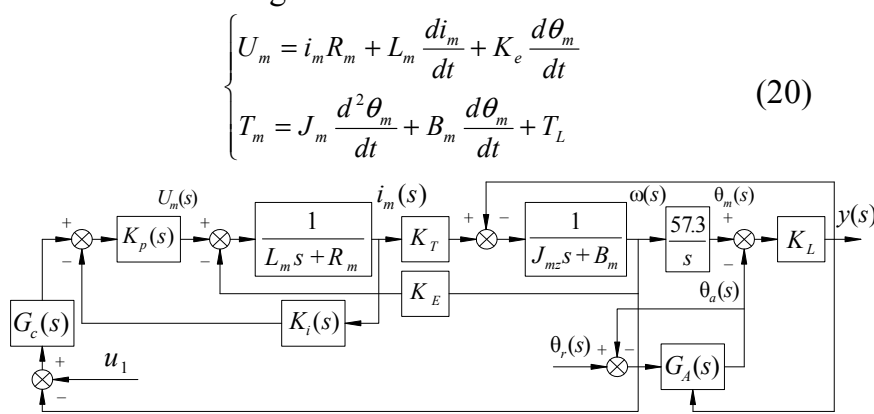

Fig.6. Dynamic model of the ELS

where $u_{1}$ is the output of the controller, $K_{p}$ is the power amplification coefficient, $L_{m}$ is the inductance of the motor winding, $R_{m}$ is the motor winding resistance, $K_{T}$ is motor torque coefficient, $J_{m z}$ is the sum of moment of inertia of motor and load, $B_{m}$ is the motor damping coefficient, $K_{E}$ is the back EMF constant of motor, $\theta_{\mathrm{r}}(s)$ is the desired rotary angle of the rudder. $\theta_{\mathrm{a}}(s)$ is the actual output of rudder, $y$ is the load torque, $\omega(s)$ is the rotation angular speed, $i_{m}$ is the motor current.All units of the parameters are international units. $K_{i}(s)$ is feedback part of the current detection, $G_{c}(s)$ is transfer function of speed adjuster based on PI control, $G_{A}(s)$ is the whole position transfer function of the rudder.

Here, we are concerned about the output angle of rudder, and the angle is measured by the angle sensor, we suppose that the output of the rudder is nearly equal to the input of the rudder, i.e. $\theta_{a}(s) \approx \theta_{r}(s)$.

\section{INTEGRATED CONTROL SYSTEM}

Fig.7 is the integrated control system based on single neuron PID adaptive and modified repetitive control.

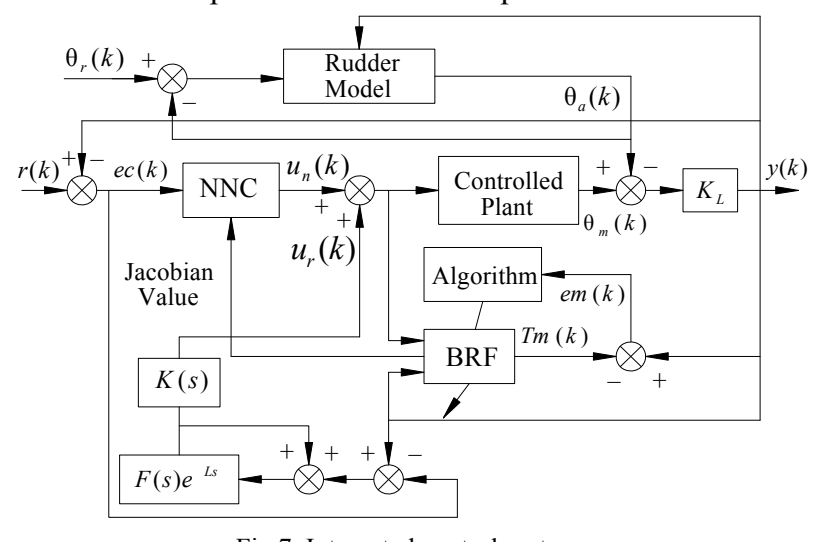

Fig.7. Integrated control system

It can also be seen from it that the output of integrated controller is made up of two parts, one is the output of the single neuron PID adaptive controller, the other is the output of repetitive controller, i.e., $u(k)=u_{n}(k)+u_{r}(k)$. When the system is stably running and the tracking error is small, the repetitive controller plays a dominant role, and after several cycles' compensation, the steady state precision can be improved quickly. When the system has a sudden change of load or other disturbance, the tracking error accordingly increases, because of the time delay with a period, the output of repetitive controller can not change immediately, so here the single neuron PID adaptive controller plays a dominant role so that it can make the system keep on good running state.

\section{SySTEM SIMULATION AND ANALYSIS}

The controlled plant is designed as three-loop control structure, namely speed loop and current loop are composed of servo unit and torque motor, the torque loop is composed of the proposed integrated controller.

The main parameters of the ELS are as below

$J_{m z}=0.35 \mathrm{kgm}^{2}, \quad L_{m}=26 \mathrm{mH}, \quad R_{m}=1.41 \Omega$, $B_{m}=0.022 \mathrm{Nm} / \mathrm{s} / \mathrm{rad}, \quad K_{E}=17.5 \mathrm{~V} / \mathrm{rad} / \mathrm{s}, \quad K_{T}=30.4 \mathrm{Nm} / \mathrm{A}, \quad K_{L}$ $=36.5 \mathrm{Nm} / \mathrm{deg}$, sampling time is $1 \mathrm{~ms}$.

Speed adjuster part is designed combining with estimation and experiment and system bandwidth based on 
proportion and integration with suitable value, the discrete equation in Fig. 6 can be presented as below

$$
\begin{gathered}
y(k)=2.8905 y(k-1)-2.8377 y(k-2)+0.9472 y(k-3)+ \\
0.0003 u(k-1)+0.0011 u(k-2)-0.0003 u(k-3)
\end{gathered}
$$

In order to evaluate the performance of the proposed control system effectively, supposing the ELS is working with low frequency and small torque, for example, the frequency $f$ is $1 \mathrm{~Hz}$ and the input (unit: $\mathrm{Nm}$ ) can be described as follows

$$
r(t)=10 \sin (2 \pi f t)
$$

The RBFFF structure is 3-6-1, and the network input $X=[u(k), y(k), y(k-1)], \eta_{1}=\eta_{2}=\eta_{3}=0.5, \eta=0.28, \alpha=0.05$ , the weight value is random number within the range [$1,+1]$. Moreover, after being trained off-line many times, the stable weight value and centre vector of all the nodes and basis width vector are gained and choose them as the system initial values.

Fig. 8 shows the torque tracking result with the input under, and the red line represents the system output and the blue line represents the input, and the two curves coincide basically in respective figure, which implies that the system achieves the very good tracking to the given signal.

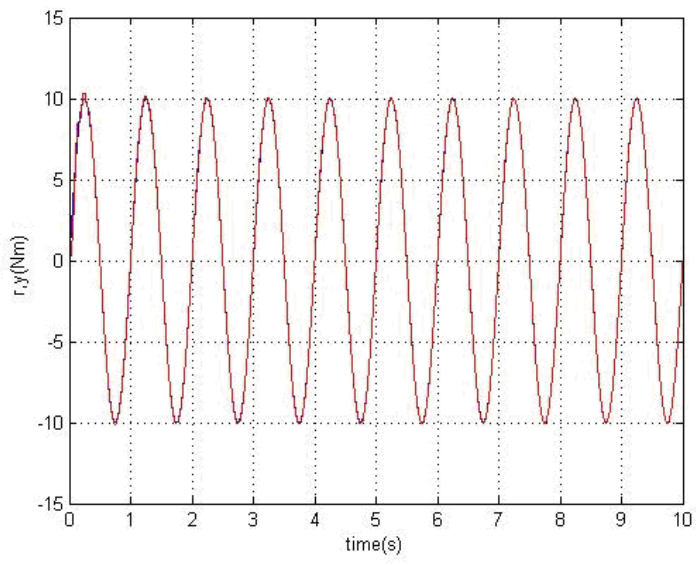

Fig.8. Torque tracking result with the input

The system error is shown in Fig.9, thus it can be seen that after several period compensation, the maximum error decreases obviously to the 8.3 percent of the maximum error of the first period. Through Fourier analysis, the amplitude distinction is $1.6 \%$, and the phase lag is $0.7^{\circ}$.

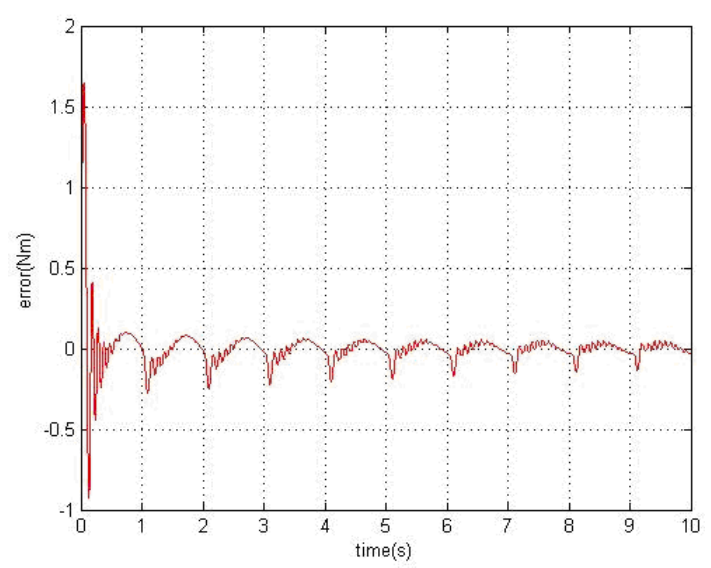

Fig.9. Tracking error

Fig.10 shows the tracking error after adding a unit step disturbance signal to the system at 1.5 second in the second period, and it is evident that the disturbance signal can be quickly and effectively restrained by the functions of selflearning and self-adapting. Hence, the integrated control strategy can effectively guarantee the good dynamic-steady state performance and strong robustness.

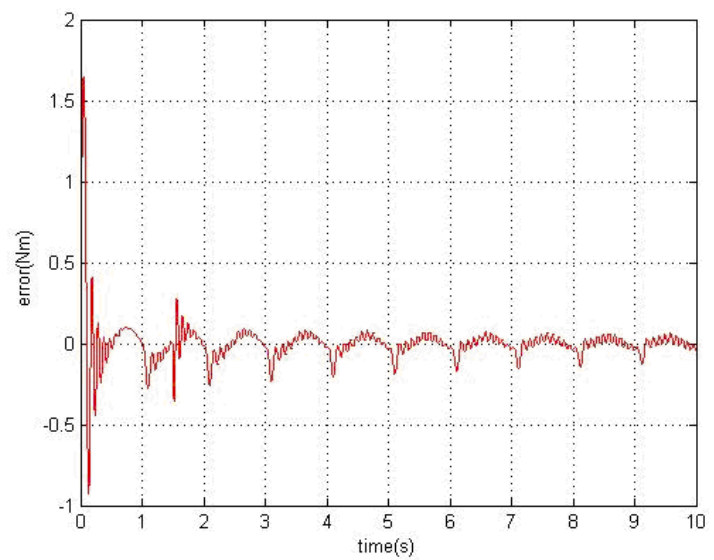

Fig.10. Tracking error after adding a unit step disturbance signal

\section{CONCLUSIONS}

In order to further improve the reliability and control precision of the ELS, a integrated control strategy is proposed based on the single neuron PID adaptive and modified repetitive controller, the approach makes full use of their own merits such as the functions of self-learning and self-adapting of neuron network and learning and memory functions of repetitive controller, which can effectively improve the performance of the torque control system. The simulation results show that under real time demand the system error can quickly converge and the surplus torque is effectively restrained, so the torque control precision is high and also disturbances can be effectively eliminated. Therefore, both the algorithm and the results are satisfying. 


\section{REFERENCES}

[1] Xingjian Wang,Shaoping Wang,Xiaodong Wang,"Electrical Load Simulator Based on Velocity-Loop Compensation and Improved Fuzzy-PID," Proceeding of the ISIE,2009,pp.238-243.

[2] Bo Yang,Zhe Wang,"Hybrid Control based on a Novel Fast Convergence and High Precision CMAC for Electric Loading System," Proceeding of the ICEMI, Vol.3,2009,pp.2573-2577.

[3] Jianxia Zhang, Yong Yang, Dezhi Xu,et al, "Single neuron adaptive PID control for AC variable-speed system,"Electric Machines and Control, Vol.11, No.2, March 2007, pp.131-137.

[4] Chao Yang, Huaichun Zhou, Xuheng Zhuo,"PID cascade control system of power plant combustion with RBF net work self-tuning," Journal of Huazhong University of Science \& Technology(Natural Science Edition), Vol.36, No.7, July 2008, pp.57-59.

[5] Changliang Xia, Jie Xiu, "RBF ANN nonlinear prediction model based adaptive PID control of switched reluctance motor," Proceeding of the CSEE, Vol.27, No.3, January 2007, pp.57-62.

[6] Yao Jin, Dejie Yu, Xiaolin Song, "Optimal Adaptive Control for Vehicle Active suspension based on single neuron PID,"China Mechanical Engineering,Vol.17, No.18, September 2006, pp.19721974.

[7] Hara S,Yamamoto Y,Omata T,et al,"Repetitive control system:a new type servo system for periodic exogenous signals," IEEE Transactions On Automatic Control, Vol.33, No,7, July 1988, pp.659-668.

[8] Mingzhu Li, Zhongyi He,Yan Xing, "Analysis and design of repetitive controlled inverter system with high dynamic performance,'Power Electronics and Motion Control Conference,2006. IPEMC 2006, Vol.3, August 2006, pp.1-5.

[9] Xin Wang,Dongzhu Feng, Haoyu Hu,"Design and Experiment of Rudder Load Simulator for High Dynamic Aircraft," Proceeding of the ICIEA,2009,pp.2573-2577. 